\title{
Kentron
}

Revue pluridisciplinaire du monde antique

$24 \mid 2008$

L'imaginaire utopique, de ses sources dans le monde grec à la Renaissance

\section{Utopie et religion chez Évhémère}

\section{Sébastien Montanari}

\section{(2) OpenEdition}

Journals

Édition électronique

URL : http://journals.openedition.org/kentron/1612

DOI : 10.4000/kentron.1612

ISSN : 2264-1459

Éditeur

Presses universitaires de Caen

\section{Édition imprimée}

Date de publication : 1 décembre 2008

Pagination : 79-104

ISBN : 978-2-84133-322-6

ISSN : 0765-0590

Référence électronique

Sébastien Montanari, « Utopie et religion chez Évhémère », Kentron [En ligne], 24 | 2008, mis en ligne le 13 mars 2018, consulté le 17 novembre 2020. URL : http://journals.openedition.org/kentron/1612 ;

DOI : https://doi.org/10.4000/kentron. 1612

\section{(c) (i) (9)}

Kentron is licensed under a Creative Commons Attribution-NonCommercial-NoDerivatives 3.0 International License. 


\section{UTOPIE ET RELIGION CHEZ ÉVHÉMÈRE}

C'est essentiellement grâce à deux résumés de Diodore de Sicile, dont l'un est transmis par Eusèbe, et à une traduction latine d'Ennius, dont des fragments sont conservés chez Lactance, que nous pouvons nous faire une idée relativement précise de l'Inscription sacrée d'Évhémère, ouvrage du début de la période hellénistique, aujourd'hui perdu. Évhémère racontait qu'il avait été envoyé en mission par le roi de Macédoine Cassandre, et que, parti des côtes de l'Arabie heureuse, il avait navi-

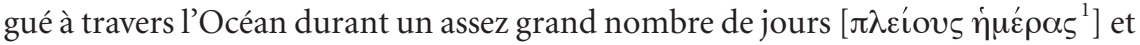
avait finalement découvert plusieurs îles, dont deux occupent une place importante dans le résumé de Diodore : l'île Sacrée et surtout l'île de Panchaïe, depuis laquelle on peut voir se dessiner dans le lointain, en direction de l'est, les côtes de l'Inde, en-

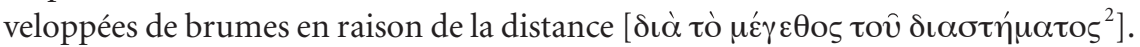
Situées aux limites du monde connu, l'Arabie heureuse et l'Inde ${ }^{3}$, pour lesquelles l'intérêt s'était accru à la suite des conquêtes d'Alexandre, apparaissaient, dans l'imaginaire grec, comme des régions empreintes de merveilleux, caractérisées par leur richesse hors du commun, leur fertilité extraordinaire, l'aspect et les mœurs parfois étranges de leurs habitants ou encore les bizarreries de leur faune et de leur flore. Aussi, en situant son récit au-delà de ces territoires des confins, au beau milieu de l'Océan, dans des îles pratiquement coupées de tout contact avec le monde extérieur $^{4}$, Évhémère donnait-il immédiatement à sa Panchaïe les traits d'un monde

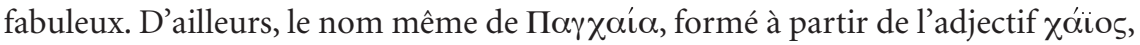
signifie étymologiquement «la très vénérable», «la très noble » ${ }^{5}$, et a donc déjà à lui seul une valeur programmatique, affirmant par avance le caractère exceptionnel de l'île. Et effectivement, la Panchaïe telle que nous la connaissons par Diodore se

1. Diod. VI, 1,4 .

2. Diod. V, $42,4$.

3. Cf. notamment Auberger 2001 et Mund-Dopchie \& Vanbaelen 1989.

4. L'île Sacrée fait en partie exception, car elle pratique le commerce de l'encens (cf. Diod. V, 42, 3). Il $\mathrm{y}$ a donc un processus de gradation dans l'éloignement.

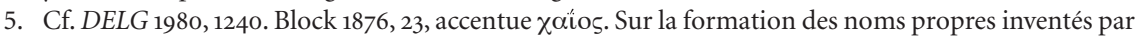
Évhémère, cf. Ferguson 1975, 105. 
distingue d'abord par sa fertilité, sa beauté, sa richesse, la grande diversité de sa flore, et ses habitants, les Panchaïens, dont la société est très organisée et dirigée par des prêtres, paraissent ${ }^{6}$ vivre dans un monde heureux, à l'abri des besoins grâce à la générosité de la nature et à un partage égalitaire des récoltes. Ainsi, même si l'affirmation devra être confirmée et éventuellement nuancée par une analyse de détail, l'ouvrage d'Évhémère semble bien pouvoir être qualifié de récit utopique ${ }^{7}$, quelle que soit l'étymologie retenue pour le terme « utopie ${ }^{8}$, le «non lieu» ou le «bon lieu», puisque s'y trouvent décrits une société et un monde à la fois imaginaires et, au moins à première vue, idéaux.

Mais la I $\varepsilon \rho \dot{\alpha}$ 'A $v \alpha \gamma \rho \alpha \phi \eta ́$ ne se limitait pas à la simple description d'un univers à caractère utopique. Évhémère rapportait en effet que se trouvait dans l'île un important sanctuaire dédié à Zeus Triphylios ${ }^{9}$, et que dans le temple, érigé par Zeus lui-même, se trouvait une stèle d'or sur laquelle étaient gravés les exploits des principaux dieux, à commencer par Ouranos, Cronos et Zeus ${ }^{10}$; ce sont très probablement les inscriptions de la stèle qui donnaient son titre au récit d'Évhémère ${ }^{11}$ et la plus grande partie de l'ouvrage devait être ainsi consacrée à l'exposé du contenu de ces inscriptions ${ }^{12}$. Cicéron, qui a pu connaître l'Inscription sacrée à travers la traduction d'Ennius, explique dans son De natura deorum qu'Évhémère avait indiqué

6. Nous employons le modalisateur "paraissent » car Diodore ne dit nulle part explicitement que les Panchaïens sont heureux.

7. La Panchaïe est qualifiée régulièrement de terre d'utopie : cf. par exemple Thraede 1965, 878; Mossé 1969; Zumschlinge 1976, 25; Bichler 1984, 187; Holzberg 1996; Peterson 2001, 4; Winiarczyk 2002, 23-25. Dans la mesure où il s'agit d'un concept moderne, on doit le manier avec précaution.

8. Le terme « utopie » vient du latin moderne utopia. C'est en effet le nom qu'en 1516 Thomas More a donné à une île de son invention dans son ouvrage De optimo reipublicae statu deque noua insula Utopia. Étymologiquement, le mot utopia signifie « qui n'existe nulle part » et est formé à partir du grec тóлos (lieu) précédé de la négation oủ. Le Dictionnaire historique de la langue française (A. Rey (dir.), Paris, Dictionnaires Le Robert, 1992, p. 2203-2204) définit l'utopie dans son sens premier comme un "pays imaginaire où un gouvernement idéal règne sur un peuple heureux ». L'idée de bonheur est en effet importante dans le concept d'utopie et, de fait, le neveu même de Thomas More proposait une variante étymologique du mot, en le supposant formé non plus à partir de la négation oú mais de l'adverbe $\boldsymbol{\varepsilon}$ (bon) (cf. sur ce dernier point Perterson 2001, 4) : l'utopie ne désigne donc pas seulement le «lieu de nulle part», mais aussi le «bon lieu », le lieu idéal.

9. Diod. V, 42, 7 .

10. Diod. V, 46,8 et VI, 1,5 .

11. C'est ce que suggère le vocabulaire employé par Diodore $(V, 46,8)$, qui, pour décrire le contenu de

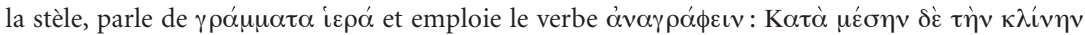

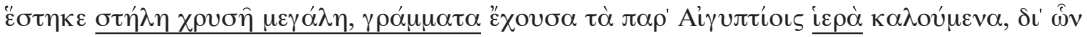

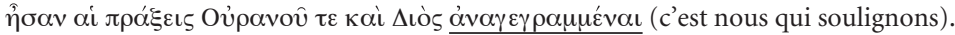

12. On sait par Athénée (XIV, 658 E-F) que l'ouvrage comptait au moins trois livres, et que, dans le troisième, il était question de Cadmos. On peut imaginer, même s'il ne s'agit que d'une hypothèse, que le premier livre présentait la Panchaïe, et les suivants le contenu de la stèle. Le deuxième livre pourrait avoir porté sur les dieux, et le troisième sur les héros. 
«les morts et les sépultures des dieux» ${ }^{13}$. De fait, les fragments que nous avons conservés d'Ennius, et qui tous reproduisent des passages de la stèle ${ }^{14}$, présentent la généalogie des premiers dieux, Ciel, Saturne et Jupiter, et les décrivent comme les premiers souverains de la terre; conquérant d'un immense empire, Jupiter est peint en roi civilisateur ${ }^{15}$ et bienfaiteur, dispensateur des lois ${ }^{16}$, garant de la justice ${ }^{17}$, protecteur des inventeurs ${ }^{18}$, qui, à la fin de sa vie, partage son royaume entre ses parents et amis, puis abandonne la vie qu'il menait en Crète et part rejoindre les dieux ${ }^{19}$. EnniusÉvhémère ajoute que l'on peut voir son tombeau en Crète, à Cnossos ${ }^{20}$. Ainsi, les grands dieux de la Grèce étaient présentés comme des hommes qui, pour leur puissance et leurs bienfaits, avaient été proclamés dieux. Jupiter fut le premier à rendre un culte à son grand-père ${ }^{21}$ et fut même, à l'occasion d'alliances stratégiques avec des rois locaux, sinon l'initiateur, du moins le propagateur de son propre culte ${ }^{22}$, en se faisant construire partout où il se rendait des temples en son honneur. Par le biais de son prétendu voyage en Panchaïe, Évhémère revisitait donc la mythologie traditionnelle, en en proposant une version historicisée quelque peu iconoclaste: les dieux étaient des hommes puissants divinisés, les premiers souverains de l'histoire.

L’objet de la présente étude est de tenter de comprendre le rôle que pouvait avoir la description de la Panchaïe dans un ouvrage qui avait avant tout, semble-t-il, pour but de relater l'histoire des grands dieux de la Grèce, dépeints comme des humains divinisés. La tâche n'est pas aisée en raison de l'aspect fragmentaire de nos sources, mais on peut espérer arriver à un résultat satisfaisant dès lors qu'on ne considère pas simplement la Panchaïe comme un monde utopique à étudier pour lui-même, mais comme le cadre narratif d'un ouvrage dont la portée est plus vaste: la Panchaïe

13. Cicéron, De natura deorum, I, 119: Ab Euhemero autem et mortes et sepulturae demonstrantur deorum.

14. Cf. Lactance, Institutions divines, I, 14, 6 .

15. En particulier, il met fin au cannibalisme (Lact., ibid., I, 13, 2).

16. Lact., ibid., I, 13, $2 ; \mathrm{I}, 11,45$.

17. Lact., ibid., I, 11, 35 .

18. Lact., ibid.

19. Lact., ibid., I, 11, 46 : Aetate pessum acta in Creta uitam commutauit et ad deos abiit.

20. Lact., ibid.

21. Lact., ibid., I, 11, 63 et Diod. VI, 1, 9. Le passage d'Ennius, qu'il n'y a pas lieu de discuter ici, est quelque peu ambigu en raison de l'homonymie entre Caelus, grand-père de Jupiter, et caelus désignant le ciel. On peut penser que Jupiter instituait un culte conjoint à son grand-père et au ciel.

22. Cf. Lact., ibid., I, 22, 21-26. Le texte d'Ennius fait la part belle à l'instauration par Jupiter lui-même de sa propre apothéose, et il n'est pas question, dans les passages conservés par Lactance, d'une divinisation spontanée de Jupiter par les différents peuples en raison de ses bienfaits. L'existence dans l'ouvrage d'Évhémère d'une telle reconnaissance spontanée du caractère divin de Zeus n'est cependant pas à exclure; le texte d'Eusèbe (Diodore VI, 1,9 ) semble même plutôt inviter à une telle interpréta-

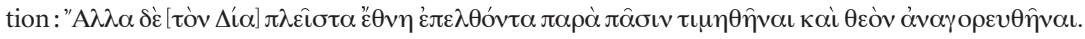


est d'abord le lieu de la découverte des inscriptions sacrées du temple de Zeus ${ }^{23}$. Il paraît cohérent d'appréhender de manière dialectique la relation entre les deux parties de l'ouvrage que sont le récit du voyage en Panchaïe et le compte rendu du contenu de la stèle. Dans cette perspective, il nous faudra à la fois mettre en évidence les caractéristiques propres des îles décrites par Évhémère et tenter d'analyser le rôle que pouvait avoir cet univers utopique dans l'ensemble de l'ouvrage.

\section{Richesse et fertilité de la Panchaiie}

\section{Une nature généreuse}

Ce qui frappe d'abord, à la lecture de la description de l'île Sacrée comme de celle de Panchaïe, c'est la générosité de la nature. Pour chaque type de plante évoquée, c'est le registre de l'hyperbole qui est systématiquement employé afin de souligner l'extraordinaire abondance qui règne sur l'île. L'île Sacrée produit ainsi une

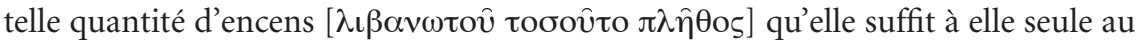

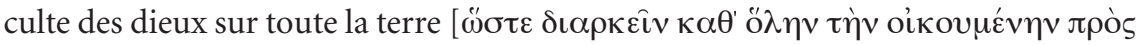
$\tau \grave{\alpha} \varsigma \tau \hat{\omega} v \theta \varepsilon \hat{\omega} v \tau \tau \mu \alpha \dot{s}]^{24}$. L'île compte même un certain nombre de terres à la qualité exceptionnelle, puisque Diodore ajoute qu'en terrain favorable, les habitants obtiennent deux récoltes de myrrhe par $a^{25}$. Dans l'île de Panchaïe, les fruits poussent sur

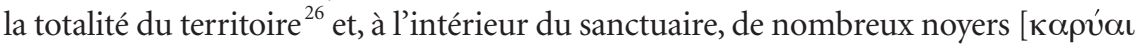

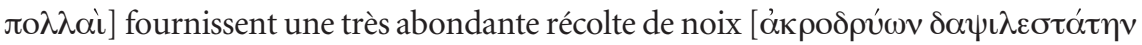

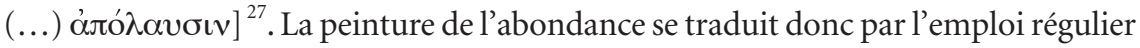
de l'adjectif $\pi \mathrm{o} \lambda u \varsigma^{28}$, du nom $\pi \lambda \hat{\eta} \theta 0 \varsigma^{29}$, d'adjectifs désignant une totalité comme ö $\lambda \circ \varsigma^{30}$ et $\pi \hat{\alpha} \varsigma^{31}$, ou encore d'adjectifs au superlatif: les vignes du sanctuaire fournis-

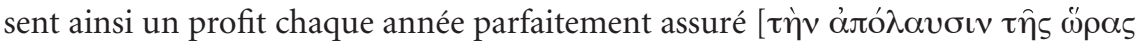

23. Le risque inhérent à toute étude partielle de l'ouvrage, ne s'intéressant qu'à la description de la Panchaïe ou au contenu de la stèle est, aussi intéressante qu'elle soit, de livrer une vision quelque peu faussée de l'ensemble.

24. Diod. $\mathrm{V}, 41,5$.

25. Diod. $V, 41,7$.

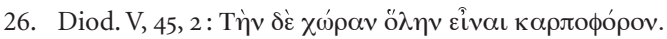

27. Diod. $\mathrm{V}, 43,3$.

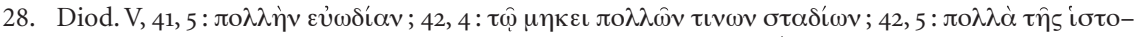
 $\lambda$ oí $[\ldots] \pi \mathrm{o} \lambda \lambda \alpha i^{\prime}$.

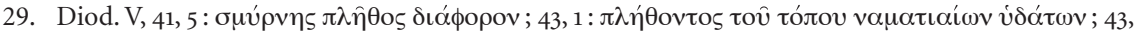

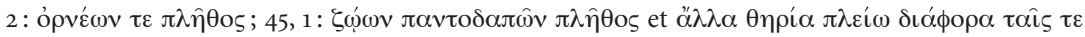

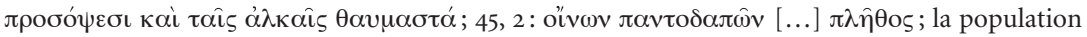
est également nombreuse : 43, $2: \pi \lambda \hat{\eta} \theta 0 \varsigma \alpha \dot{\alpha} v \delta \rho \hat{v}$.

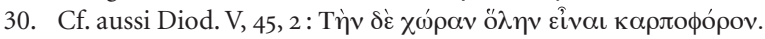

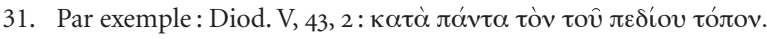




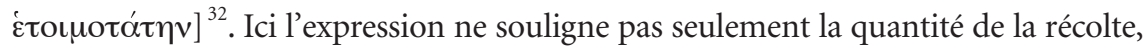
mais aussi sa parfaite régularité. La nature se montre constamment généreuse au

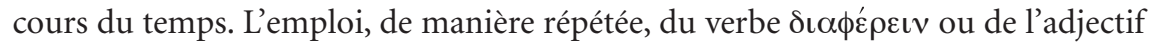
et de l'adverbe équivalents, marque également, dans tous les domaines, le caractère exceptionnel de l'île. Les troncs de palmiers qui poussent dans le sanctuaire sont ainsi

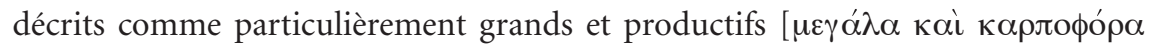
$\delta \iota \alpha \phi \varepsilon \rho o ́ v \tau \omega \varsigma]^{33}$.

\section{Diversité de la faune et de la flore}

Une autre manière, pour Évhémère, de rendre compte de la prospérité inhabituelle de la Panchaïe et de l'abondance qui y règne est d'insister sur la diversité de la faune et de la flore: plantes et animaux ne sont pas seulement nombreux ou de grandes tailles ${ }^{34}$, mais d'espèces très variées. C'est la répétition régulière de l'adjectif

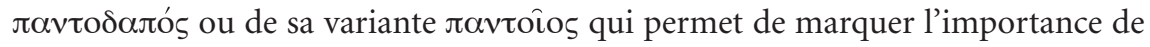
cette diversité. La Panchaïe possède ainsi des parfums de toutes natures [ $\tau \hat{\omega} v \nu \alpha^{\prime} \lambda \lambda \omega v$

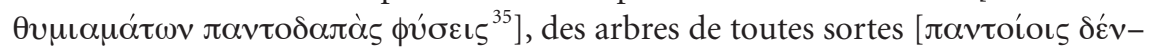

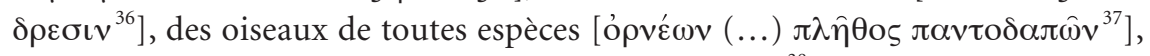

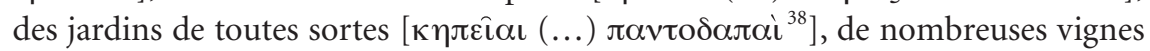

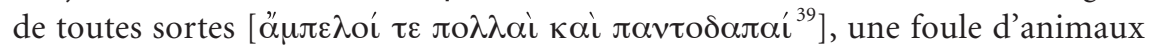

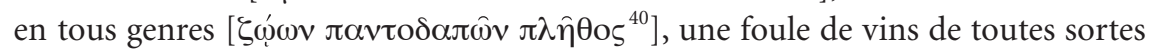
[öv $\left.\omega v \pi \alpha v \tau \operatorname{co\delta } \alpha \pi \hat{\omega} v(\ldots) \pi \lambda \hat{\eta} \theta 0 \varsigma^{41}\right]$. Cette diversité très nettement soulignée de la faune et de la flore est là encore l'expression de l'incroyable fertilité de la Panchaïe.

\section{Abondance de l'or et de l'argent}

La générosité de la terre de Panchaïe ne se manifeste pas, par ailleurs, uniquement dans sa flore généreuse, mais aussi dans la richesse minière de ses sous-sols et

32. Diod. V, 43, 3 .

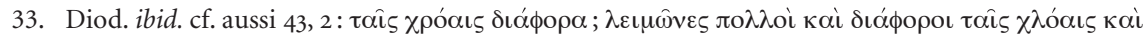

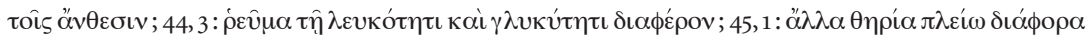

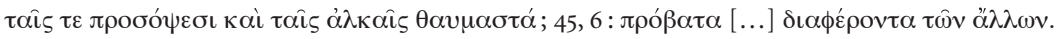

34. Pour la faune, Diodore précise en V, 45, 1 que la Panchaïe compte, entre autres animaux, de nombreux éléphants.

35. Diod. V, 41, 5 .

36. Diod. V, $43,1$.

37. Diod. V, 43, 2.

38. Diod. ibid.

39. Diod. V, 43, 3 .

40. Diod. V, 45, 1 .

41. Diod. V, 45, 2. 
dans le nombre important de ses sources. «Le Pays», dit Diodore, «compte d'abondantes mines d'or, d'argent, de cuivre, d'étain et de fer » ${ }^{42}$. La mention régulière de l'or suffit à suggérer sa profusion dans l'île: les femmes et les hommes se parent d'or ${ }^{43}$, les prêtres se coiffent de mitres tissues d'or et portent des bijoux en or ${ }^{44}$. Ils offrent aux dieux d'importantes offrandes d'or et d'argent ${ }^{45}$. Les portes du temple sont ornées d'or, d'argent et d'ivoire ${ }^{46}$; le lit du dieu est en or massif ${ }^{47}$, et la stèle qui fait le récit des exploits des dieux est également en or ${ }^{48}$. Utilisé comme comparant, l'or investit même le champ symbolique pour souligner la richesse incroyable de l'île : la fleur de l'arbre à encens est ainsi qualifiée de $\chi \rho v \sigma o \varepsilon ı \delta \varepsilon ́ \varsigma^{49}$. Si l'on en croit les témoignages de Pline l'Ancien, d'Hygin et de Cassiodore ${ }^{50}$, Évhémère avait même fait de la Panchaïe le pays de l'or par excellence, puisqu'elle était censée être, grâce à Éaque, le fils de Jupiter, le lieu de la première découverte du métal précieux. Cette abondance de l'or en Panchaïe joue plusieurs rôles : elle souligne la richesse et donc l'importance du sanctuaire de Zeus; elle est ensuite conforme à la légendaire richesse de l'Orient, de la Perse à l'Inde, qui est régulièrement décrite dans la littérature grecque, d'Hérodote aux historiens d'Alexandre en passant par Ctésias de Cnide; enfin et surtout, elle permet de dessiner une sorte d'anti-Grèce, un monde idéal pourvu de ce qui est rare chez les Grecs.

\section{Richesse en eau de la Panchaïe}

La mention des nombreuses sources que comptent le sanctuaire et la plaine environnante relève exactement du même procédé. La plaine regorge de sources

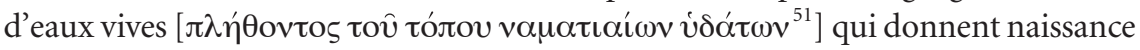
à une végétation foisonnante. Symbole de vie, précieuse en Grèce pour sa rareté, l'eau abonde dans l'île, en particulier dans le sanctuaire, qui possède une source dont le débit est extraordinaire: «Près de l'enceinte sacrée », écrit Diodore, «jaillit

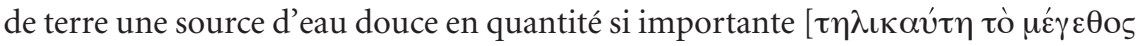
$\pi \eta \gamma \eta ́$ ] qu'il en naît un fleuve navigable » ${ }^{52}$. Si l'on songe qu'en Grèce, les lits des

\footnotetext{
42. Diod. V, 46, 4 (toutes les citations en français de Diodore sont des traductions personnelles).

43. Diod. $\mathrm{V}, 45,6$.

44. Diod. $V, 46,2$.

45. Diod. V, 46,5 et VI, 1,4 .

46. Diod. V, 46,6

47. Diod. ibid.

48. Diod. V, 46, 8 et VI, 1,5 .

49. Diod. $\mathrm{V}, 41,6$.

50. Pline l'Ancien, Histoire naturelle, VII, 197; Hygin, Fables, 274, 4: Aeacus Iouis filius in Panchaia in monte Taso aurum primus inuenit; Cassiodore, Divers, IV, 34, 3 .

51. Diod. V, 43, 1 .

52. Diod. $\mathrm{V}, 43,2$.
} 
cours d'eau étaient généralement asséchés en été, le contraste entre le monde grec et le paysage panchaïen est évident. Ici, pour souligner l'abondance, Évhémère ne joue plus seulement sur le procédé d'accumulation consistant à souligner le nombre important de sources, mais sur celui d'amplification : la quantité d'eau qui surgit de la terre est impressionnante. Et selon le même principe, les arbres arrosés par les cours d'eau sont d'une hauteur extraordinaire ${ }^{53}$. Un peu plus loin dans le texte, Diodore livre par ailleurs une seconde description de la source qui la dote de toutes

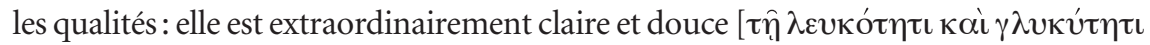
$\left.\delta \iota \alpha \phi \varepsilon^{\prime} \rho v^{54}\right]$, ce qui souligne sa pureté, et la boire est bénéfique pour la santé ${ }^{55}$. À la fois source et fleuve en un même lieu, à la fois une et multiple puisqu'elle se divise en de nombreux bras ${ }^{56}$, la source du sanctuaire totalise ainsi toutes les fonctions qui peuvent être celles d'un cours d'eau, incarnant une forme de perfection idéale : elle est navigable, elle arrose les cultures, son eau est non seulement propre à la consommation, mais elle a des vertus médicinales, sa localisation dans le sanctuaire, qui plus est à proximité du temple ${ }^{57}$, lui confère aussi une dimension religieuse. Même si Diodore n'en dit rien, son exceptionnelle pureté la prédispose à jouer un rôle dans les rites de purification. Enfin, le nom du fleuve, «eau du soleil» [i் $\lambda$ íov

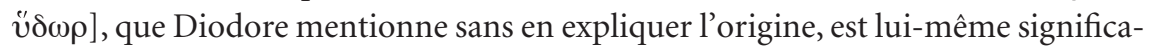
tif et parait suggérer une nouvelle fois le caractère extraordinaire du cours d'eau. Une telle appellation peut d'abord renvoyer concrètement aux reflets brillants du

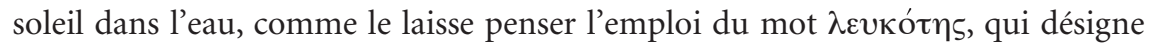

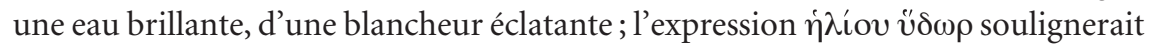

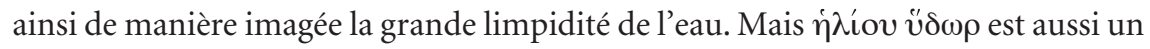
oxymore qui allie deux contraires inconciliables, l'eau et le feu : l'eau du soleil, c'est le fleuve que n'assèche pas la chaleur du soleil. C'est d'ailleurs à l'ombre des grands arbres arrosés par les eaux du fleuve que les habitants viennent se protéger de la chaleur de l'été ${ }^{58}$. Enfin, dans la mesure où il est question à plusieurs reprises d'un culte des astres dans l'ouvrage d'Évhémère, la source du sanctuaire voit aussi par son nom son lien au sacré renforcé ${ }^{59}$. Le nom « eau du soleil» traduit donc bien sous ses différents aspects la nature idéale du fleuve.

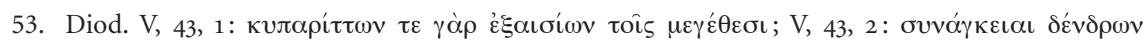

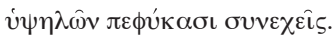

54. Diod. $\mathrm{V}, 44,3$.

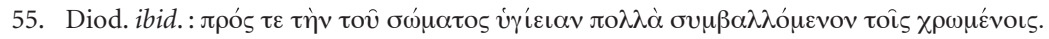

56. Diod. $\mathrm{V}, 43,2$.

57. Cf. Diod. V, 44, 2-3.

58. Diod. V, 43, 2.

59. Cf. Diod. VI, 1, 6 et Lact. Inst. I, 11, 63. 


\section{Beauté du paysage}

Le paysage panchaïen se caractérise enfin par son exceptionnelle beauté. À pro-

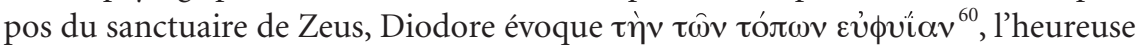
nature ou l'heureux aspect des lieux, ce qui renvoie à la fois à l'idée de fertilité et de beauté. Ainsi, les vignes qui poussent dans le sanctuaire ne se contentent pas de fournir d'abondantes récoltes, mais par leurs mille entrelacs, elles agrémentent le

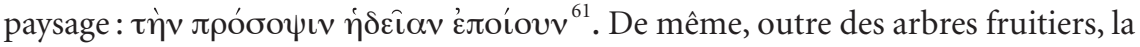
plaine environnant le sanctuaire compte encore toutes sortes d'arbres propres à ré-

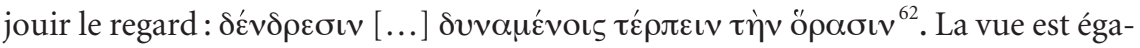
lement conviée dans la description de la faune, puisqu'on trouve dans le sanctuaire

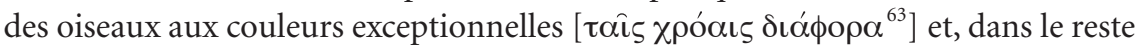

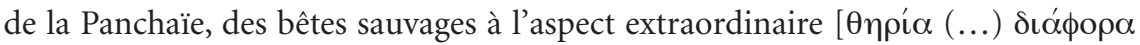
$\tau \alpha i \varsigma$ (...) $\left.\pi \rho \circ \sigma o ́ \psi \varepsilon \sigma_{\iota}{ }^{64}\right]$. Plus généralement, la Panchaïe est un monde offert au plaisir des sens : l'ouïe est sollicitée à travers le chant empli de charme des oiseaux

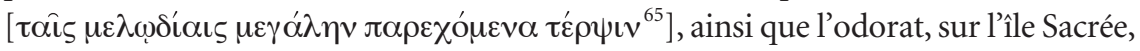
puisque les parfums de toutes sortes $\mathrm{y}$ « répandent leurs bonnes odeurs à profu-

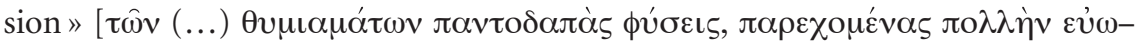
$\left.\delta \delta^{\prime} \alpha v^{66}\right]$. Fertilité extraordinaire, abondance, beauté, plaisir des sens, la Panchaïe, et plus particulièrement le sanctuaire de Zeus, possèdent tous les éléments du locus amœnus, du paysage agréable et idéal.

\section{La présence de la divinité}

\section{Des précédents : Homère et Platon}

Cette générosité et cette beauté de la nature doivent être comprises comme le signe de la présence du divin en ce pays du bout du monde. Déjà chez Homère, les Éthiopiens, qui vivent dans l'abondance, aux marges du monde, et qui ont conservé leur nature primitive, reçoivent la visite régulière des dieux avec lesquels ils festoient longuement ${ }^{67}$. Et toujours dans l'Odyssée, les lieux marqués par la présence du divin sont d'une beauté et d'une richesse exceptionnelles; c'est le cas par exemple des

\footnotetext{
60. Diod. V, 42, 7 .

61. Diod. V, 43, 3 .

62. Diod. V, 43, 1 .

63. Diod. V, 43, 2.

64. Diod. V, 45, 1.

65. Diod. V, 43, 2.

66. Diod. V, 41, 5 .

67. Cf. par exemple Homère, Odyssée, I, 22-26. Cette commensalité des hommes et des dieux est le souvenir d'une époque ancienne où hommes et dieux vivaient côte à côte.
} 
abords de la caverne de la nymphe Calypso, au livre $\mathrm{V}^{68}$. On découvre là aussi un paysage enchanteur: des sources qui traversent des prairies en fleur, une vigne fleurie de grappes, un bois qui sert de refuge à de nombreux oiseaux, des arbres odorants. Là encore les principaux sens sont conviés : dans sa caverne, Calypso chante de sa belle voix, et le dieu Hermès, avant d'entrer dans la caverne pour rencontrer la nymphe, s'arrête pour admirer l'endroit ${ }^{69}$. «Dès l'abord en ces lieux ", dit Homère, «il n'est pas d'Immortel qui n'aurait eu les yeux charmés, l'âme ravie » ${ }^{70}$. Le paysage habité ou fréquenté par les dieux est ainsi à l'image de leur perfection et le plaisir que ressent Hermès en le contemplant est le reflet de l'harmonie qui existe entre sa nature divine et celle du paysage. On pourrait multiplier les exemples de paysages divins dans la littérature grecque : on songera par exemple, toujours dans l'Odyssée, à la description du jardin du roi des Phéaciens, Alkinoos ${ }^{71}$. Les Phéaciens sont un peuple des confins et ils sont proches des dieux; et les extrêmes fertilité et beauté du jardin royal soulignent cette proximité avec les dieux : «Tels étaient les présents magnifiques des dieux au roi Alkinoos », conclut Homère à la fin de sa description ${ }^{72}$. Mentionnons encore, plus tard, dans le Critias de Platon, la description de l'île de l'Atlantide ${ }^{73}$, dont la richesse, la fertilité et la beauté extraordinaires sont explicitement présentées comme l'œuvre du dieu Poséidon:

Ce fut Poséidon lui-même qui embellit l'île centrale et il n'y eut point de peine, étant Dieu. Il fit jaillir de dessous le sol deux sources d'eau, l'une chaude, l'autre froide et pousser sur la terre des plantes nourricières de toute sorte, en suffisance ${ }^{74}$.

Les habitants tirent ainsi leur immense prospérité de la présence du dieu, qui a reçu l'Atlantide en partage ${ }^{75}$, et de sa bienveillance à leur égard.

\section{Paysage et divinité}

Évhémère, dans sa description de la Panchaïe, reprend donc cette représentation, traditionnelle depuis Homère, du paysage imprégné de la présence du sacré.

68. Voir les vers 55 à 77 .

69. Hom. Od. V, 75-76.

70. Hom. Od. V, 73-74 (nous reprenons, dans les passages cités en français d'Homère, la traduction de

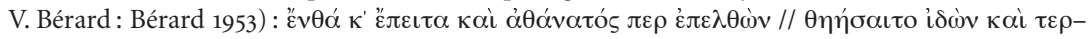

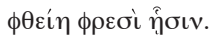

71. Hom. Od. VII, 112-132.

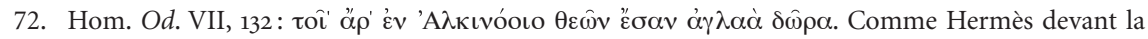
grotte de Calypso, c'est cette fois Ulysse qui, par ailleurs, reste admiratif devant le jardin d'Alkinoos, et dans l'un et l'autre cas, ce sont justement les deux mêmes vers, au changement de nom près, qui traduisent l'admiration du dieu et du héros (cf. Od. V, 75-76 et VII, 133-134).

73. Voir Platon, Critias, 113-119.

74. Plat. Critias 113 e.

75. Plat. Critias $113 \mathrm{c}$. 
De fait, autrefois, la Panchaïe était fréquentée par ceux qui allaient devenir les grands dieux de la mythologie, à commencer par Ouranos, qui, lorsqu'il régnait sur le monde,

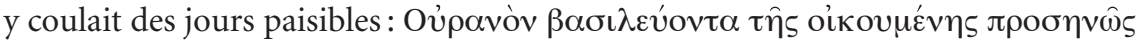

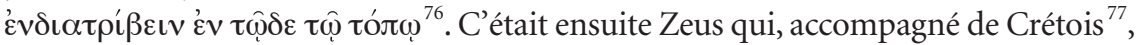
s'était rendu un temps en Panchaïe, où il avait élevé un autel à son aïeul Ouranos ${ }^{78}$, s'était fait construire un temple ${ }^{79}$, et était l'auteur des inscriptions racontant ses exploits et ceux d'Ouranos ${ }^{80}$. Évhémère soulignait d'ailleurs explicitement le lien entre la majesté du paysage et la présence des dieux: «Par la magnificence divine qui s’offre

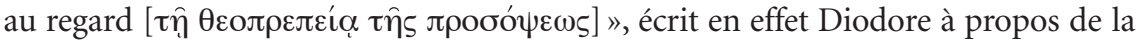
plaine environnant le sanctuaire, «la plaine paraît digne des dieux qui l'habitent

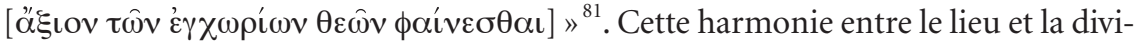
nité se révèle aussi dans l'architecture magnifique du temple; les adjectifs qui servent

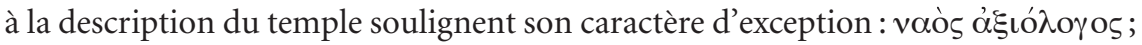

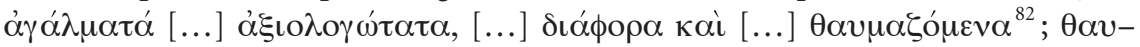

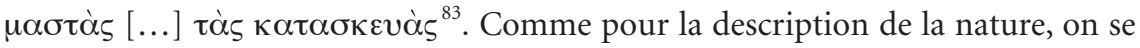
place d'emblée dans le registre de l'exceptionnel, de l'admirable. Évhémère ne semble pas décrire un temple parmi d'autres, mais plutôt le temple par excellence, concentrant en lui toutes les qualités possibles. Diodore insiste d'abord sur son caractère imposant: sa longueur de deux plèthres le rend, pour la taille, comparable au temple de Zeus à Olympie ${ }^{84}$, ses colonnes sont à la fois hautes et imposantes [Kíođı $\delta \dot{\varepsilon}$

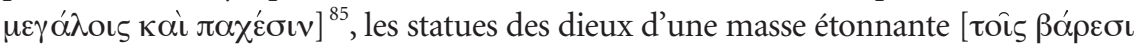
$\theta \alpha v \mu \alpha \zeta o ́ \mu \varepsilon v \alpha]^{86}$. Le temple et les statues sont ensuite décrits par Diodore comme

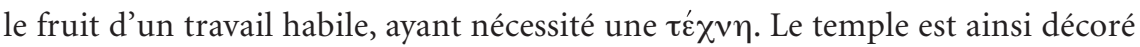

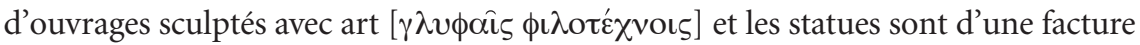

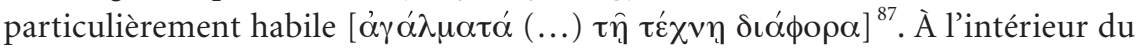

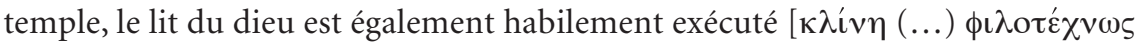

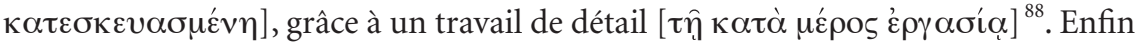

76. Diod. V, 44,6 .

77. Diod. $V, 46,3$.

78. Diod. VI, 1, 9.

79. Diod. $\mathrm{V}, 46,3$.

80. Diod. V, 46,3 et 8.

81. Diod. V, 43, 2.

82. Diod. V, 44, 1.

83. Diod. V, 46, 6 .

84. Cf. Zumschlinge 1976, 34 .

85. Diod. V, 44, 1.

86. Diod. ibid.

87. Diod. ibid.

88. Diod. V, 46, 6. Remarquons également que dans le passage de Diodore transmis par Eusèbe, l'auteur de la Bibliothèque mentionne rapidement les curiosités de la Panchaïe dont il a rendu compte dans 
le temple se caractérise par sa grande richesse: outre les nombreuses offrandes d'or et d'argent, les magnifiques ornements d'argent, d'or et d'ivoire, la table et la stèle en or que nous avons déjà évoqués plus haut, mentionnons encore les sculptures en bois de thuya de la porte du temple, un bois rare et précieux. Devant le temple, une avenue longue de quatre stades et bordée de grandes statues de bronze $[\chi \alpha \lambda \kappa \varepsilon \hat{\imath} \alpha$ $\mu \varepsilon \gamma \alpha \dot{\lambda} \lambda \alpha]^{89}$ ajoute encore à la somptuosité et à la richesse de l'ensemble. La beauté, la richesse, le caractère imposant du temple et des statues des dieux font écho au paysage environnant, dans lequel ils s'insèrent ainsi parfaitement.

Tant par la description de sa nature magnifique, généreuse et fertile que par celle de l'architecture extraordinaire de son sanctuaire, la Panchaïe se révèle donc être un monde divin. Même si, à l'époque du voyage d'Évhémère, les dieux ne sont plus physiquement présents dans l'île - Ennius rapporte en effet que le tombeau de Zeus se trouve en Crète, à Cnossos ${ }^{90}$-, la majesté des lieux prolonge à travers le temps la mémoire de cette présence ancienne et le culte que les prêtres continuent de rendre à Zeus, depuis l'époque où il a lui-même fait construire son propre temple, maintient également vivant le souvenir du séjour des Olympiens.

\section{Société et transmission du culte des dieux}

\section{Piété des Panchaïens}

La société panchaïenne, telle qu'elle s'est constituée à la suite de l'arrivée de Zeus dans l'île, semble en effet pour l'essentiel construite autour du culte de Zeus et la perpétuation du souvenir des dieux. Dans le résumé de Diodore transmis par Eusèbe, où le peuple de Panchaïe est brièvement évoqué, les seules informations données par Diodore sont d'ordre religieux : Évhémère, raconte-t-il, a vu

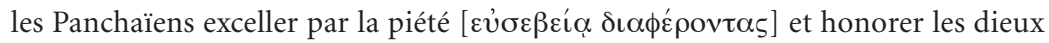

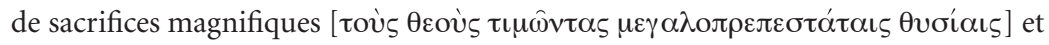

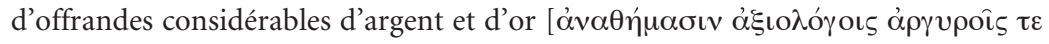
кaì $\chi \rho$ pvoîs] $]^{91}$.

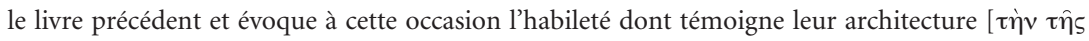

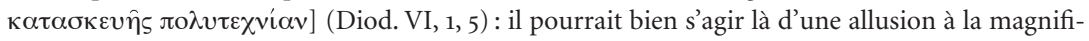
cence du temple de Zeus.

89. Cf. Diod. V, 44, 3 .

90. Lact. Inst. I, 11, 46. Quant à Ouranos, il est, toujours selon Ennius, in Oceania mortuum et in oppido Aulacia sepultum (Lact. Inst. I, 11,65). La ville d'Aulacia n'est pas connue par ailleurs, et l'Océanie semble être un pays inventé par Évhémère que l'étymologie invite à situer au bord de l'Océan, probablement encore conçu comme fleuve du monde. Parmi les peuples étrangers habitant la Panchaie, Diodore mentionne les Océanites, qu'on peut, d'après leur nom, aisément supposer originaires d'Océanie (cf. Diod. V, 42, 5). L'Océanie est donc un pays étranger à la Panchaïe, et comme Zeus, Ouranos n'est pas enterré en territoire panchaïen.

91. Diod. VI, 1,4 . 
Il est significatif que dans ce très court résumé qui devait aller immédiatement à l'essentiel, la seule caractéristique donnée de la population ait été son extrême piété: il s'agissait évidemment d'un trait saillant de la société panchaïenne. Le vocabulaire

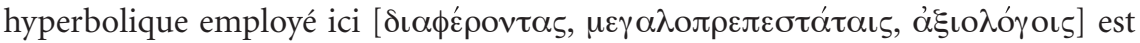
sensiblement le même que pour la description de la Panchaïe : à un paysage ou à un monde marqué par le divin correspond une société à la piété hors du commun. Diodore conclut d'ailleurs le passage en précisant que l'île est consacrée aux dieux

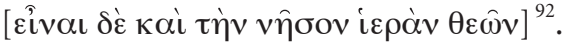

\section{Le rôle des prêtres}

Le signe le plus visible de l'importance que la société panchaïenne accorde à la religion est la place qu'y occupent les prêtres. D'origine crétoise, ils sont les descendants des hommes qui avaient accompagné le souverain de la terre, Zeus, jusqu'en Panchaïe, où ils s'étaient installés ${ }^{93}$. Quoique Diodore ne soit pas très explicite sur ce point, on peut penser que leurs ancêtres n'étaient pas prêtres à l'origine, mais qu'en tant que compagnons de Zeus, ils s'étaient vu confier par ce dernier l'administration de son propre culte et du sanctuaire, après la construction du temple. Les prêtres constituent la classe dirigeante de l'île, ont autorité sur tout et gèrent les affaires

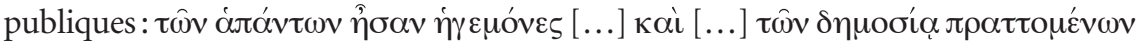
кúpıo ${ }^{94}$. Mais leur principale activité reste le culte des dieux:

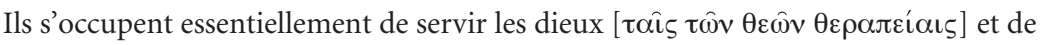

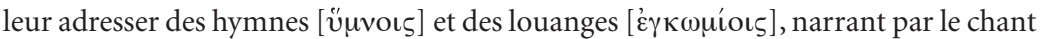

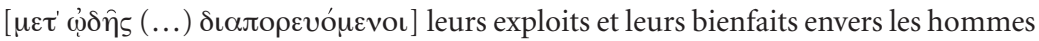

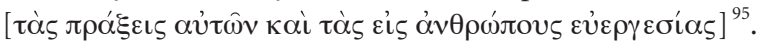

La mention qui est faite ici du contenu des hymnes est particulièrement intéressante puisqu'elle met en évidence ce que sont les principales qualités de la divinité : un dieu accomplit de hauts faits et se montre bienfaisant envers l'humanité. Au-delà des rites sacrificiels, le culte consiste dès lors à rendre grâce aux dieux en se remémorant leurs actes et leurs bienfaits : les prêtres de Panchaïe sont des sortes d'aèdes dont les chants sont à la fois louanges à la gloire des dieux et mémoire d'un passé ancestral. Ce rôle de conservation de la mémoire de la geste divine dévolu aux prêtres est souligné à plusieurs reprises dans le texte. C'est ainsi que sur la montagne appelée «Olympe Triphylios » et anciennement nommée «Siège d'Ouranos " ${ }^{96}$, les

92. Diod. VI, 1,5 .

93. Diod. $\mathrm{V}, 46,3$.

94. Diod. $\mathrm{V}, 45,4$

95. Diod. V, 46, 2.

96. Diod. V, 44, 6. 


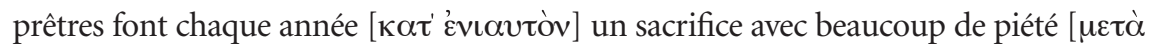
$\left.\pi 0 \lambda \lambda \eta \bar{\jmath} \varsigma \dot{\alpha} \gamma v \varepsilon \varepsilon_{\alpha \varsigma}\right]^{97}$ : ce faisant, ils perpétuent le culte instauré par Zeus à son grandpère sur cette montagne ${ }^{98}$, depuis laquelle Ouranos aimait observer les astres ${ }^{99}$. Les prêtres sont aussi décrits comme très attachés aux liens anciens d'amitié qu'ils ont conservés avec les Crétois, souvenir de l'époque de Zeus. Cette tradition s'est trans-

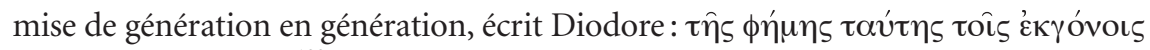
$\pi \alpha \rho \alpha \delta ı \delta o \mu \varepsilon ́ v \eta \varsigma \alpha \varepsilon^{\prime} i^{100}$. Enfin et surtout, les prêtres sont capables de prouver l'authenticité de cette tradition grâce à des inscriptions qu'ils disent avoir été faites par

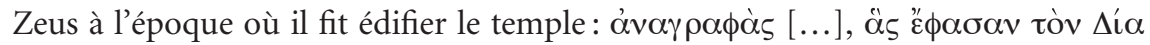

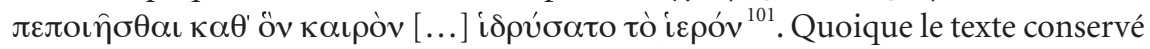
par Diodore ne l'indique pas explicitement, ces inscriptions ne font sans doute qu'un avec celles gravées sur la fameuse stèle d'or narrant les exploits d'Ouranos et de Zeus ${ }^{102}$. Ainsi, de génération en génération, les prêtres sont les gardiens d'une vérité ancienne sur les dieux, conservée sous forme d'inscriptions dans le temple de Zeus.

\section{De Platon à Évhémère, les prêtres gardiens de la mémoire du passé}

Cette représentation chez Évhémère des prêtres en détenteurs de la mémoire du passé n'est pas nouvelle. Dans le Timée de Platon, ce sont des prêtres égyptiens qui ont conservé par écrit dans leurs temples le souvenir de l'époque de l'ancienne Athènes et du royaume de l'Atlantide: "S'il s'est accompli quelque chose de beau, de grand ou de remarquable à tout autre égard », explique l'un des prêtres à Solon, « tout cela est ici par écrit, depuis l'antiquité, dans les temples, et la mémoire en a été sauvée ${ }^{103}$. La civilisation égyptienne était réputée chez les Grecs pour son ancienneté $^{104}$, et c'est sans doute en grande partie pour cette raison que, dans le récit de Platon, le sage grec Solon apprenait l'histoire de l'Athènes des premiers temps auprès de prêtres de Saïs. Chez Évhémère, les prêtres de Panchaïe occupent dans la

\footnotetext{
97. Diod. V, 44, 7 .

98. Cf. Lact. Inst. I, 11, 63.

99. Diod. $\mathrm{V}, 44,6$.

100. Diod. V, 46, 3 .

101. Diod. ibid.

102. Deux éléments permettent un tel rapprochement: d'une part, le récit de l'arrivée des Crétois sur l'île a toute sa place sur la stèle, puisqu'il constitue l'un des épisodes des aventures de Zeus, et d'autre part Lactance, dans ses Institutions divines, raconte que c'est Jupiter lui-même qui écrivit le détail de ses exploits, ce qui nous invite à mettre sur le même plan le texte de la stèle et ces inscriptions montrées par les prêtres, elles aussi rédigées par Zeus (cf. Lact. Inst. I, 11, 33: auream columnam positam esse ab ipso Ioue titulus indicabat, in qua columnam sua gesta perscripsit).

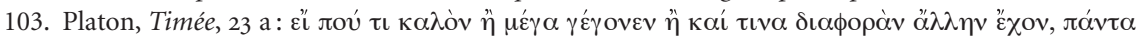

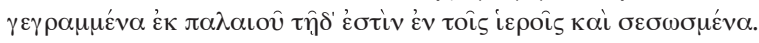

104. Cf. déjà chez Hérodote (II, 143) le récit de la rencontre entre Hécatée et des prêtres de la Thèbes d'Égypte, qui prouvent à l'historien grec que le monde est beaucoup plus ancien qu'il ne le croit.
} 
fiction un rôle strictement équivalent à celui rempli par les prêtres égyptiens dans le Timée : ils sont les seuls à détenir la mémoire d'un passé tellement ancien qu’il a été oublié par le reste du monde. Chez Platon, le prêtre de Saïs explique que les populations qui peuplent la terre sont régulièrement anéanties par le feu ou par l'eau, et que les rares survivants de ces cataclysmes, généralement illettrés et ignorants, perdent en même temps que l'écriture la mémoire de leur passé et doivent ainsi régulièrement repartir de zéro. Mais la géographie particulière de l'Égypte, qui, par son fleuve, est protégée des sécheresses et, par ses montagnes, des déluges, a permis à la civilisation égyptienne de survivre chaque fois à l'eau et au feu, et donc à ses prêtres, principaux détenteurs de l'écriture, de devenir la mémoire du monde. Pour Platon, une telle conception cyclique du temps et de l'histoire des hommes présentait un intérêt évident : la parole du prêtre de Saïs assurait une parfaite légitimité à son exposé sur l'Atlantide, en lui conférant une ancienneté plus grande que tous les récits connus jusque-là chez les Grecs ${ }^{105}$. Non seulement la fiction recevait ainsi un parfum d'authenticité, mais elle devait avoir la prééminence sur tout autre récit en raison de son ancienneté plus grande. Le principe devait être le même chez Évhémère. Le texte de la stèle présentait une nouvelle vision des dieux et de la mythologie, et la seule manière de donner une légitimité à ces inscriptions était de leur attribuer une ancienneté et une authenticité plus grandes que les récits traditionnels. Or les prêtres de Panchaïe rapportaient que le texte de la stèle avait été composé par Zeus lui-même, puis complété par Hermès pour ce qui concernait les exploits d'Artémis et d'Apollon ${ }^{106}$. Des inscriptions écrites par les dieux eux-mêmes: on ne pouvait pas rêver texte à la fois plus authentique et plus ancien!

\section{La Panchaïe, ou comment faire des inscriptions du temple un texte authentique}

\section{Une société ancienne}

Pour garantir l'ancienneté de la stèle, la Panchaïe devait elle-même apparaître comme un monde ancien. Le premier signe de l'antiquité de l'île résidait d'ailleurs

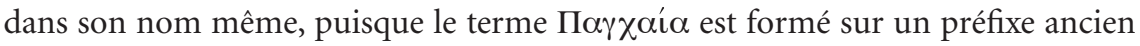
$\pi \alpha v$ - et sur l'adjectif dorien archaïque $\chi \alpha$ áio $^{107}{ }^{107}$. Dans le résumé livré par Eusèbe, Diodore rapporte également, sans donner davantage de détails, que l'île compte bien

\footnotetext{
105. Platon avait d'ailleurs d'autant mieux assuré le succès de son récit que, par un habile retournement, l'ancienne Athènes était ensuite donnée par le prêtre pour antérieure de 1000 ans à la cité de Saïs. In fine, la Grèce reprenait le pas sur l'Égypte pour l'ancienneté, ce qui ne devait pas déplaire au lecteur athénien, fier de son autochtonie.

106. Cf. Diod. V, 46, 8.

107. Cf. note 5 .
} 


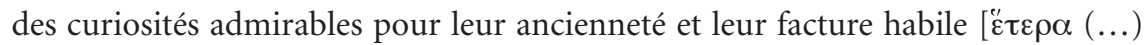

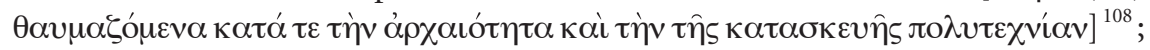
il s'agit très certainement d'une allusion au temple de Zeus, décrit au livre $\mathrm{V}$ de la

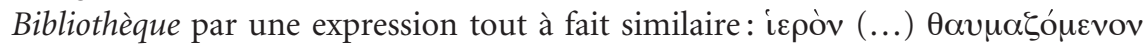

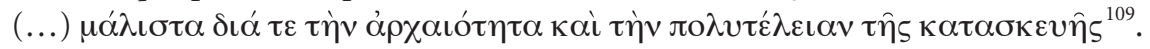
Il était normal que le temple qui abritait l'antique stèle fût lui-même très ancien. L'ancienneté de la stèle se trouve également soulignée par la nature des signes qui y sont gravés : elle porte, écrit Didore, «des caractères que chez les Égyptiens on ap-

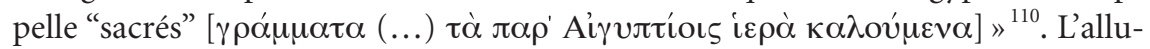
sion à des hiéroglyphes, indéchiffrables pour le commun des mortels, et à l'Égypte, civilisation ancienne par excellence, ne marque pas simplement la stèle du sceau du sacré, mais invite aussi à faire remonter les inscriptions du temple à la plus haute antiquité.

Au-delà du temple lui-même et de la stèle, plusieurs traits de la société panchaïenne concourent encore à faire de l'île un monde ancien. Diodore explique par exemple que les hommes utilisent des chars dans les combats, suivant l'usage anti-

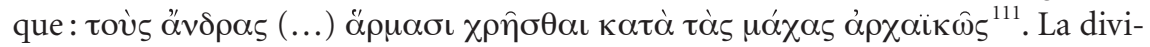
sion de la société en trois classes, prêtres et artisans d'une part, paysans d'autre part, et guerriers et bergers enfin, suggère aussi l'ancienneté de l'organisation sociale de la Panchaïe. Aristote, dans la Politique, attribuait l'invention de la division du corps civique en classes aux sociétés égyptiennes et crétoises et la faisait remonter à une époque très reculée, au temps de Sésostris pour l'Égypte et à celui du roi légendaire Minos pour la Crète ${ }^{112}$. Les Grecs connaissaient d'ailleurs depuis longtemps l'existence du principe de la division en classes chez les Égyptiens puisqu'on le trouve déjà mentionné chez Hérodote, qui divise la population égyptienne en sept catégories, les prêtres occupant la première place au sein de la société ${ }^{113}$. Dans le Timée de Platon, le prêtre égyptien évoque lui aussi la division en classes de la société égyptienne et en attribue même l'origine à l'ancienne Athènes. Platon mentionne ainsi plusieurs catégories de population - les prêtres, les artisans, les bergers, les veneurs,

108. Diod. VI, 1,5 .

109. Diod. V, $42,7$.

110. Diod. V, 46, 8. Le texte transmis par Eusèbe (Diod. VI, 1, 5) parle de son côté de lettres panchaïen-

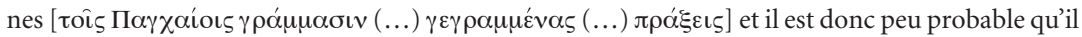
faille comprendre dans l'expression utilisée par Diodore que la stèle était écrite en égyptien; c'est moins de la langue qu'il est question ici que de la nature spéciale des caractères utilisés dans le domaine du sacré chez les Égyptiens : le texte de la stèle est rédigé dans une écriture hiéroglyphique.

111. Diod. V, 45, 3. Les habitants de l'ancienne Atlantide combattent également sur des chars (cf. Plat. Critias $119 \mathrm{a}-\mathrm{b})$.

112. Cf. Aristote, Politique, VII, X, 1-2 [1329 a 40-1329 b].

113. Cf. Hérodote II, $164 ; 167-168$. 
les laboureurs et les combattants ${ }^{114}$ - qui toutes, mis à part les veneurs, se retrouvent chez Évhémère. Sans entrer pour l'instant dans le détail des catégories retenues par Évhémère et de leur répartition, on peut donc admettre que la division de la population en classes, avec sans doute une référence implicite à l'Égypte, visait d'abord à donner à la société panchaïenne une coloration ancienne.

\section{De l'histoire à l'éternel présent d'une société immuable}

Ce n'était pas, cependant, le seul avantage que pouvait présenter pour Évhémère la division en classes. Il ne suffisait pas que la vérité révélée par le texte de la stèle s'imposât au lecteur par son ancienneté, il fallait encore pouvoir expliquer comment cette vérité avait pu survivre au passage du temps sur le sol de Panchaïe, tandis que manifestement, elle avait été oubliée du reste du monde ${ }^{115}$. Au temps des dieux, même si le texte transmis par Diodore ne permet pas d'établir une chronologie précise de l'antique histoire panchaïenne, la Panchaïe a participé au processus historique en même temps que le reste du monde. Le premier signe de ce passage du temps se mesure d'abord à travers les visites successives des dieux: Ouranos, d'abord, puis Zeus, qui élève un autel à son grand-père ${ }^{116}$, Hermès, qui ajoute sur la stèle les exploits d'Artémis et d'Apollon ${ }^{117}$. D’une génération à l'autre, des changements ont lieu, puisque par exemple la montagne depuis laquelle Ouranos obser-

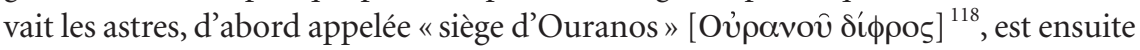

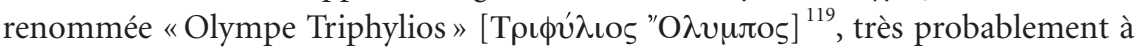

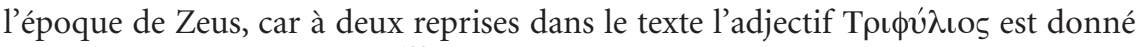
comme une épiclèse de Zeus ${ }^{120}$. L'̂̂le de Panchaïe a par ailleurs connu plusieurs vagues d'immigration, et au peuple autochtone des Panchaïens, sont venus s'ajouter d'autres peuples, Océanites et Doïens, déjà présents à l'arrivée de Zeus ${ }^{121}$. À une

114. Cf. Plat. Timée 24 a-b.

115. Aucun des fragments conservés ne dit explicitement que cette vérité avait été oubliée du reste du monde, ce qui n'est pas obligatoirement dû à une omission de Diodore, mais peut très bien correspondre à un choix d'Évhémère lui-même, qui n'a pas, dans ce cas, jugé nécessaire d'expliciter ce qui paraissait évident: si la véritable histoire de Zeus - qui concerne l'ensemble du monde, puisqu'il avait conquis toute la terre habitée - n'est conservée qu'en Panchaïe, c'est que le reste du monde l'a, sinon oubliée, du moins déformée. C'est d'ailleurs ce que rapportait Philon de Biblos dans un récit probablement inspiré d'Évhémère, dont des passages nous ont été transmis par Eusèbe de Césarée (Préparation évangélique, I, IX, 22-30).

116. Cf. Diod. VI, 1, 9.

117. Cf. Diod. V, 46, 8.

118. Diod. V, 44, 6.

119. Diod. ibid.

120. Les habitants de la ville de Panara sont en effet appelés «suppliants de Zeus Triphylios» $(V, 44,6)$ et le sanctuaire se nomme «sanctuaire de Zeus Triphylios» (V, 44, 7).

121. Ces trois premiers peuples, Panchaïens, Océanites et Doïens, sont à l'origine de l'épiclèse de Zeus Triphylios (cf. Diod. V, 44, 6), ils sont donc présents dans l'île à l'arrivée de Zeus. 
époque indéterminée, des Indiens et des Scythes ${ }^{122}$ s'installent également sur l'île, et enfin, le roi Zeus amène avec lui des Crétois, qui vont constituer la future classe dirigeante de l'île. À l'inverse, à une époque sans doute postérieure, le peuple des Doïens doit quitter l'île, exilé par Ammon, et ses cités sont détruites ${ }^{123}$. Immigration, conquête ${ }^{124}$, exil, tous ces bouleversements historiques remontent à l'époque des dieux.

Par la suite, l'histoire ne paraît plus avoir prise sur l'île. On ne sait pas si les institutions panchaïennes sont l'œuvre de Zeus, présenté par Lactance comme un législateur ${ }^{125}$, d'un autre dieu ou encore des Crétois eux-mêmes, mais elles ont contribué à placer la société de l'île hors du temps, comme figée dans un éternel présent. Les individus puissants qui ont fait l'histoire ont disparu au profit des groupes, sans qu'aucune personnalité ne se détache. Pour décrire les habitants, Diodore emploie

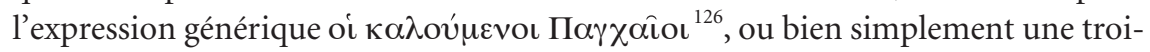
sième personne du pluriel sans sujet directement exprimé ${ }^{127}$. Lorsqu'il veut préci-

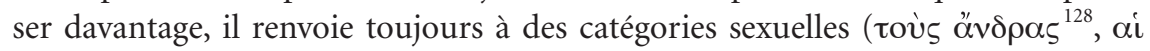

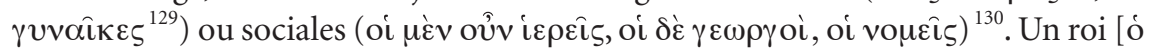
$\left.\beta \alpha \sigma i \lambda \varepsilon v u_{\zeta}\right]^{131}$ est bien mentionné sur l'île Sacrée, mais il incarne non pas une individualité particulière mais la répétition intemporelle de la fonction royale. L’impression que le temps s'est arrêté n'est pas seulement due à l'emploi répété du présent descriptif à valeur ethnologique ${ }^{132}$, utilisé aussi par Hérodote ou par les historiens d'Alexandre lorsqu'ils veulent présenter, dans une sorte d'instantané, le fonctionnement général d'une société : sur l'île de Panchaïe, la division en classes et la répartition des tâches qu'elle implique assurent manifestement la stabilité de l'État au cours du temps. Le pouvoir, définitivement entre les mains des prêtres, n'est pas l'enjeu des luttes politiques, l'un des principaux moteurs de l'histoire: les paysans se consacrent simplement au travail de la terre ${ }^{133}$, les bergers à l'élevage ${ }^{134}$; les soldats

122. Diod. V, 42, 5 .

123. Cf. Diod. V, 44, 6 .

124. L'expression «suppliant de Zeus Triphylios» $(\mathrm{V}, 44,6)$ laisse en effet penser que Zeus est arrivé en conquérant sur l'île et qu'un certain nombre de peuples se sont soumis à lui et placés sous sa protection en prenant la posture de suppliants.

125. Lact. Inst. I, 11, 45.

126. Diod. V, 42, 3 .

127. Cf. par exemple Diod. V, 42,3 et 45,6 .

128. Diod. V, 45, 3. Voir aussi V, 43, 2 et 45,6 .

129. Diod. V, $45,6$.

130. Pour ces trois exemples, se reporter successivement à Diod. V, 45, 4; 45, 4; 45, 5.

131. Diod. V, 42, 1.

132. Le texte du livre $\mathrm{V}$ de Diodore est dans l'ensemble rédigé au présent de l'indicatif, mais avec parfois des passages à l'aoriste et à l'imparfait $(44,1-2)$ ou au discours indirect libre $(45,2-3)$.

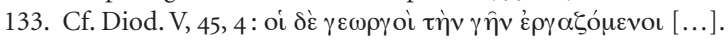

134. Cf. Diod. V, 45, 5 . 
surveillent le territoire ${ }^{135}$. Les différentes lois en vigueur semblent avoir d'abord pour but de limiter toutes les formes de désirs ou de frustrations qui pourraient nuire à la stabilité de l'île et donc à la conservation de la mémoire du passé divin. Les paysans mettent ainsi en commun les récoltes [นoù

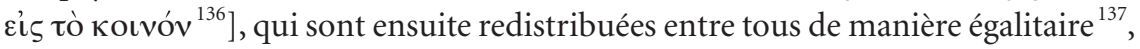
de sorte que tout le monde mange à sa faim. La monnaie ne semble pas exister sur l'île ${ }^{138}$, ce qui n'est pas seulement un signe d'archaïsme : son absence place les individus à l'abri du désir de richesse, potentiel facteur d'instabilité. Le droit de propriété est d'ailleurs strictement limité sur l'île à la possession d'une maison et d'un jardin ${ }^{139}$. Bien plus, Évhémère, sentant peut-être les limites de l'égalitarisme ${ }^{140}$, semble avoir voulu canaliser le désir de richesse pour le mettre au service de la communauté, grâce à la reconnaissance du mérite : c'est ainsi que les dix meilleurs paysans reçoivent "une gratification exceptionnelle lors du partage des récoltes »

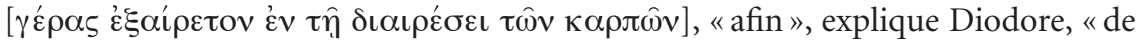

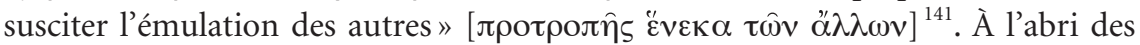
tensions de toutes sortes grâce à ces institutions immuables, la société panchaïenne peut donc vivre dans une sorte d'éternel présent, où les prêtres peuvent se consacrer essentiellement au culte des dieux ${ }^{142}$, et où le seul signe du passage du temps réside dans l'accumulation des offrandes au sein du sanctuaire: "le temps ", écrit

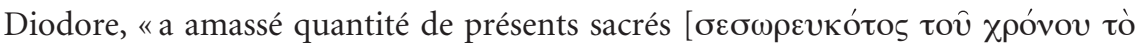

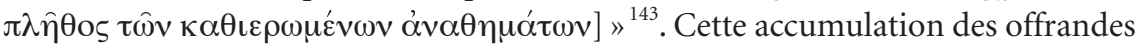
fait du sanctuaire une sorte de musée manifestant la continuité à travers le temps de l'antique piété des Panchaïens envers les hommes-dieux du passé. La société panchaïenne ayant avant tout été organisée pour la conservation fidèle de la mémoire divine, les inscriptions sacrées avaient survécu au passage du temps.

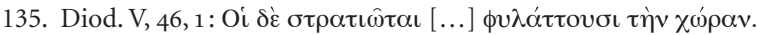

136. Diod. V, 45, 4.

137. «Les prêtres », écrit Diodore en V, 45, 5, "perçoivent la totalité des produits et des revenus, et dis-

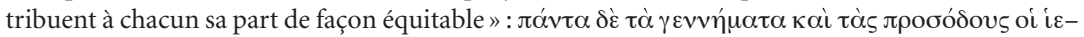

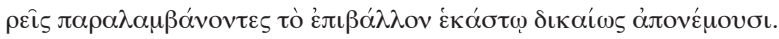

138. Il est très peu probable que cette absence soit imputable à une omission de Diodore, le système en vigueur sur l'île rendant en fait la monnaie inutile.

139. Cf. Diod. V, 45, 5.

140. On peut en effet imaginer deux limites au partage égalitaire des récoltes : il peut produire des frustrations chez ceux qui ne trouvent pas leur mérite reconnu, et il peut conduire à une baisse de productivité, chacun comptant finalement sur les autres pour assurer sa subsistance. Pour Évhémère, la communauté ne peut faire totalement abstraction du désir individuel.

141. Diod. V, 45, 4.

142. Le texte de Diodore dit explicitement qu'il s'agit de leur principale occupation $(\mathrm{V}, 46,2)$ :

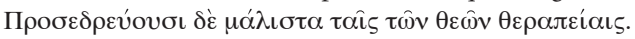

143. Diod. V, 46, 5 . 


\section{Un monde oublié}

L'organisation de la Panchaïe devait cependant encore permettre d'expliquer comment les fameuses inscriptions avaient pu rester méconnues du reste de l'humanité avant la découverte d'Évhémère. L'exigence de vraisemblance n'était plus cette fois d'ordre temporel mais spatial. D'un point de vue géographique, l'île de Panchaïe est située dans les confins ${ }^{144}$ : sa position lointaine et isolée n'en faisait pas seulement un séjour agréable pour les dieux, mais la protégeait aussi naturellement des contacts avec les autres peuples ${ }^{145}$. Après le passage des dieux, la Panchaïe avait eu son propre destin, indépendant de celui du reste de l'humanité. C'est seulement au temps du roi Cassandre, dans une époque marquée par les conquêtes d'Alexandre et par le goût de l'exploration, que la Panchaïe longtemps oubliée allait, grâce à la « découverte» d'Évhémère, réintégrer les cartes du monde. De fait, dans ce qu'on est tenté d'appeler l'archipel de Panchaï ${ }^{146}$, seule l'île Sacrée est en relation avec le monde extérieur, puisqu'elle se livre au commerce de l'encens ${ }^{147}$; cependant, le contact avec les populations hellénisées n'est pas direct: les Panchaïens, explique Diodore,

exportent l'encens et la myrrhe et les vendent à des marchands arabes, à qui d'autres marchands achètent ce genre de produits pour les transporter jusqu'en Phénicie, en Colé-Syrie ou encore en Égypte. Finalement, de ces pays, des marchands les font passer dans le monde entier ${ }^{148}$.

Remarquons d'abord qu'une telle description des circuits du commerce de l'encens rendait l'existence de l'île tout à fait plausible au lecteur : les Arabes passaient dans l'Antiquité pour être les principaux producteurs des aromates. Évhémère ne changeait donc pas radicalement la représentation que son lecteur avait de ce commerce,

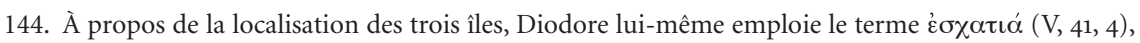

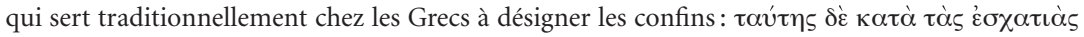

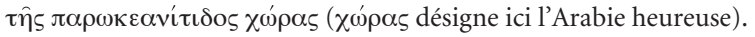

145. "Qui de bon gré parcourrait une étendue d'eau salée aussi immense?», répondait déjà Hermès à Calypso, qui constatait avec amertume qu'elle ne recevait pas souvent la visite des dieux dans son

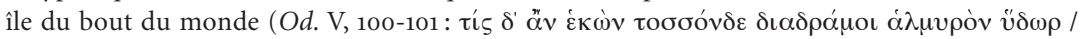

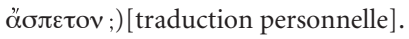

146. Peterson 2001, 30 imagine que la Panchaïe était à la fois le nom de l'archipel et de son île principale (cf. Hawai). L'idée est séduisante mais ne semble pas pouvoir être prouvée. L'expression «la Panchaïe » est en tout cas une manière commode de désigner par métonymie l'ensemble de l'univers créé par Évhémère.

147. Diod. V, 41,5 et 42,3 .

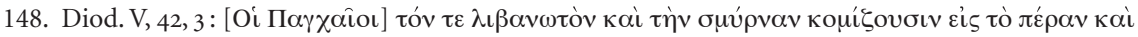

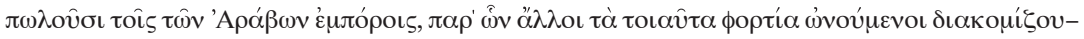

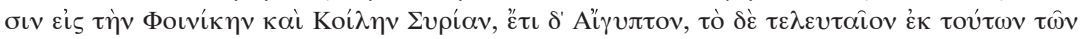

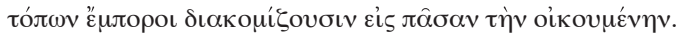


il se contentait de préciser la tradition : le principal centre de production était simplement déplacé un peu plus loin au large des côtes d'Arabie, tandis que les Arabes jouaient certes toujours un rôle capital, mais étaient en fait ravalés au rang de premiers intermédiaires de la chaîne ${ }^{149}$. Et en même temps qu'Évhémère s'efforçait de rendre vraisemblable l'existence de l'île Sacrée, la mention de trois intermédiaires différents - à commencer par les marchands arabes, étrangers à la culture grecque - avant que l'encens n'arrive entre les mains des acheteurs définitifs, expliquait que l'île Sacrée, quoique ouverte sur le monde, ait pu rester parfaitement inconnue du commun des mortels.

Avec l'île de Panchaïe, sur laquelle se trouve le sanctuaire de Zeus, une étape supplémentaire était franchie : l'île vit en pleine autarcie et paraît complètement coupée du monde. Bien qu'elle possède de l'or, de l'argent, du cuivre, de l'étain et du fer en quantité, il est interdit d'exporter ces riches métaux hors de Panchaïe :

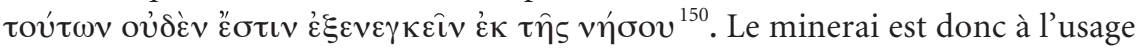
exclusif des Panchaïens et l'or sert soit à la fabrication de bijoux portés par les hommes et les femmes ${ }^{151}$, ce qui semble une allusion au luxe de l'Orient, soit au culte des dieux. Or, dans l'Antiquité, les métaux mentionnés précédemment servaient à la fabrication des monnaies; l'interdiction d'exporter le métal est donc significative: elle traduit le refus d'utiliser la monnaie dans le cadre d'échanges commerciaux, et sauf à imaginer, de manière invraisemblable, une forme de troc avec les pays étrangers, l'île de Panchaïe n'entretient aucune relation commerciale avec l'extérieur. Cette interdiction confirme d'ailleurs l'inexistence de la monnaie chez les Panchaïens. Grâce à sa richesse exceptionnelle et à la redistribution égalitaire des récoltes, la Panchaïe est une île autosuffisante, et en raison de son fonctionnement autarcique, son existence, et donc celle de la stèle, avait pu, depuis l'époque des dieux, être oubliée du reste du monde.

Évhémère avait d'ailleurs posé d'autres garde-fous en construisant son univers imaginaire. Les prêtres, qui sont par définition les principaux détenteurs de la mémoire du passé divin, ont ainsi l'interdiction absolue de quitter le sanctuaire et peuvent même être mis à mort par le premier qui les surprendrait en train de braver l'interdit ${ }^{152}$. À l'inverse, la partie centrale du sanctuaire est interdite aux profanes. La source du soleil, qui se situe dans le prolongement de la grande allée du temple, est bordée d'un quai de pierre sur quatre stades, et « jusqu'à l'extrémité du quai», indique Diodore, «le lieu est interdit d'accès à tout homme hormis aux prêtres

149. Voir déjà sur ce point Dochhorn 2000, 281: «Die Araber, traditionnel als Produzenten des Räucherwerks bekannt, werden damit zu Zwischenhändlern herabgestuft ».

150. Diod. V, 46, 4 .

151. Cf. Diod. V, $45,6$.

152. Diod. V, 46, 4. 


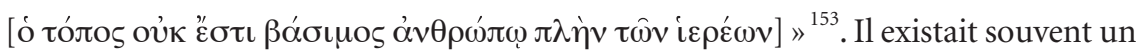
certain nombre d'interdictions au sein des sanctuaires ${ }^{154}$, mais dans le cas présent, les interdits avaient avant tout une justification narrative: ceux qui connaissaient le contenu de la stèle ne pouvaient pas quitter le territoire, et ceux qui ne le connaissaient pas ne pouvaient pas en prendre connaissance, le temple et la stèle se trouvant apparemment dans la zone interdite. Nous disons «apparemment ", car avec de telles règles, le secret était si bien gardé qu'Évhémère lui-même n'aurait pas dû avoir la possibilité d'accéder au temple ${ }^{155}$. Peut-être cette petite contradiction existaitelle déjà dans l'œuvre originale, ou bien peut-être la contradiction n'est-elle qu'une impression produite par le résumé de Diodore ${ }^{156}$. Comme nous ne connaissons pas les conditions du séjour d'Évhémère dans l'île ni les relations qu'il était censé avoir entretenues avec les prêtres, cette question ne sera jamais tranchée. Toujours est-il qu'à la lecture de l'ouvrage, tout laissait penser au lecteur qu'il était normal de ne pas avoir entendu parler auparavant des inscriptions du Zeus de Panchaïe, mais que leur nouveauté n'était qu'apparente: il s'agissait bien plutôt de la redécouverte d'un monde oublié. Le récit d'Évhémère obtenait ainsi la crédibilité recherchée.

\section{Conclusion : de l'imaginaire utopique au monde réel}

Notre hypothèse de départ selon laquelle la Panchaïe créée par Évhémère répond d'abord à une stratégie de légitimation de ses théories religieuses semble donc vérifiée par l'analyse de détail du résumé de Diodore: la Panchaïe est une île dont la fertilité, la richesse et la beauté extraordinaires apparaissent comme les vestiges, dans le présent, d'une sorte d'ancien âge d'or associé à la présence des dieux. La majesté des lieux, l'ancienneté et la piété de la société panchaïenne, tournée avant tout vers

153. Diod. V, 44, 4 .

154. Cf. Zumschlinge 1976, 37 .

155. Il ne faut en aucun cas considérer que le contenu de la stèle devait être, par principe, tenu secret. L'écrivain Évhémère avait simplement besoin d'inventer une justification au fait que la Panchaïe et la stèle avaient jusque-là échappé à la connaissance de tous. L'interdit est d'ordre narratif et non d'ordre théologique.

156. Diverses hypothèses narratives peuvent être imaginées pour lever la contradiction, mais, ne pouvant être étayées, elles n'ont qu'un intérêt tout relatif. On peut imaginer, par exemple, que la zone interdite aux profanes ne comprend pas le temple, le texte de Diodore ne permettant pas d'être absolument affirmatif, ou encore que, pour un étranger, les prêtres avaient fait une entorse à la règle... En fait, et plus simplement, il est très possible que la description de la Panchaïe ait été présentée avec le point de vue d'un narrateur omniscient, ce qui, tout en donnant une impression de neutralité et d'objectivité, dispensait justement Évhémère d'avoir à inventer dans le détail toutes sortes de justifications narratives pour maintenir la cohérence de l’ouvrage : ce qui lui importait était évidemment la description de la Panchaïe plus que le récit d'une (prétendue) expérience personnelle, dont la mise en avant ponctuelle ne servait en fait qu'à garantir la véracité de son propos. 
la conservation de la mémoire du passé divin, sont le signe de l'authenticité de la vérité religieuse transmise par les inscriptions du temple de Zeus. Malgré l'importance accordée par Évhémère à la description du système social de l'île de Panchaïe, nous ne pensons pas qu'il faille interpréter l'univers de la Panchaïe comme une utopie au sens restreint du terme, autrement dit comme un modèle politique idéal qui aurait pour vocation d'être réalisé par la suite, ou plus simplement qui viserait à provoquer la réflexion sur la société réelle du début du III $^{\mathrm{e}}$ siècle à travers la description d'une société imaginaire. Il est certes très probable que divers éléments de l'organisation sociale panchaïenne, comme le partage égalitaire des récoltes, reflètent un certain nombre de débats philosophiques de l'époque, et Claude Mossé a souligné à juste titre dans son article «Les utopies égalitaires à l'époque hellénistique ${ }^{157}$ que le III ${ }^{\mathrm{e}}$ siècle est une période de crise sociale. Cependant la société panchaïenne prise dans son ensemble n'a pas valeur de modèle comme peuvent l'avoir les sociétés imaginées par un Hippodamos de Milet ou un Phaléas de Chalcédoine. On ne doit pas perdre de vue qu'Évhémère ne livrait pas une réflexion abstraite: sa description d'une société en trois classes ne valait pas en soi, mais s'insérait dans un récit plus large à l'intérieur duquel seulement elle trouvait sa signification. $\mathrm{Ne}$ serait-ce qu'en raison des incroyables fertilité et richesse que la Panchaïe offre à ses habitants, la transposition d'une telle société dans le monde grec n'aurait pas de sens. Le choix des prêtres comme classe dirigeante ne correspond pas non plus à une exigence d'ordre politique mais narratif: l'importance de leur rôle tient surtout à l'importance dans le récit de la stèle elle-même, dont ils sont les gardiens. Évhémère ne propose d'ailleurs pas de modèle politique unique, puisque l'île Sacrée, dirigée par un roi ${ }^{158}$, semble obéir à une organisation politique différente. En Panchaïe même, la cité de Panara possède un statut de semi-autonomie et est dirigée par trois archontes nommés chaque année par les habitants ${ }^{159}$.

Cette diversité des systèmes politiques semble en fait inspirée par le monde hellénistique, où les grands royaumes coexistent avec des cités plus ou moins autonomes. La position dominante exercée par les Crétois sur les populations autochtones ou plus anciennement installées en Panchaïe n'est pas non plus sans rappeler la prééminence détenue par les couches macédoniennes et grecques sur les peuples des territoires conquis par Alexandre. Et en même temps que par sa richesse, sa fertilité et sa beauté, le paysage panchaïen présente les caractéristiques d'un monde idéal, d'autres éléments, certes moins nombreux, en révèlent les imperfections, inspirées elles aussi de la réalité contemporaine. Diodore explique par exemple que

157. Mossé 1969, 299.

158. Diod. V, 42, 1.

159. Cf. Diod. V, 42, 6. 
sur l'île Sacrée les habitants utilisent le fruit du Paliure comme remède contre la diarrhée ${ }^{160}$ : à la différence des Hyperboréens de Pindare ${ }^{161}$ ou de la race d'or d'Hésiode $^{162}$, les habitants n'échappent donc pas aux maladies, même s'ils ont les remèdes pour les soigner. En Panchaïe même, rapporte encore Diodore, « une partie du pays est victime des brigandages [ $\left.\lambda \eta \emptyset \sigma \tau \eta^{\prime} \iota \alpha\right]$ de hardis hors-la-loi qui attaquent les paysans en les prenant en embuscade ${ }^{163}$. On sait qu'à l'époque hellénistique, pillards et pirates étaient un véritable fléau pour les royaumes et les cités et représentaient une menace omniprésente pour leurs populations ${ }^{164}$. La Panchaïe n'est pas un monde parfait, mais reflète donc différentes caractéristiques de l'époque d'Évhémère. Même si par sa richesse hors du commun la Panchaïe appartient à l'imaginaire des confins, Évhémère n'a pas voulu en faire un univers complètement hétérogène à la réalité. Le récit de voyage d'Évhémère ne se donnait pas comme une fiction, et pour que le lecteur crût à l'existence de la stèle et à l'authenticité de son contenu, il ne fallait pas qu'il tînt la Panchaïe pour un monde mythique ou merveilleux. Évhémère a ainsi banni de sa description tous les éléments, pourtant traditionnels dans la représentation des confins, qui auraient pu passer pour invraisemblables. Il n'est pas question d'animaux extraordinaires ${ }^{165}$, comme le martychoras de Ctésias ou la créature imaginée par Iamboulos, à quatre yeux et quatre bouches, et dont le sang est censé pouvoir recoller tout membre sectionné ${ }^{166}$. Les hommes eux-mêmes n'ont ni capacités physiques ni espérance de vie hors du commun ${ }^{167}$. La Panchaïe est donc le résultat d'une construction complexe, qui tout en utilisant de nombreuses caractéristiques de l'imaginaire utopique - abondance, richesse, fertilité, beauté, insularité, présence du divin, éloignement aux marges du monde connu - ne s'affiche pas comme une utopie, ni en tant que «lieu de nulle part», ni totalement en tant que «bon lieu», mais se présente bien plutôt comme une extension du monde réel un

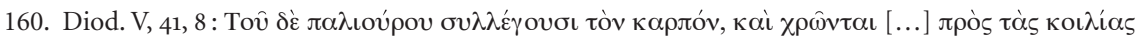

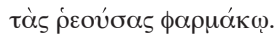

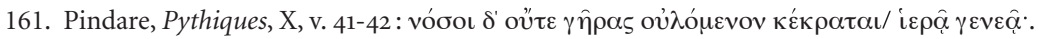

162. Cf. Hésiode, Les Travaux et les Jours, v. 113-115.

163. Diod. V, 46, 1 : "̌б

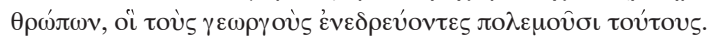

164. Cf. par exemple Chamoux 1987, 61-62; voir aussi Gabrielsen 2004.

165. Étant donné le goût de Diodore pour tout ce qui pouvait avoir un caractère étonnant, il n'est pas vraisemblable que l'absence de traits véritablement merveilleux soit due à une omission de l'auteur de la Bibliothèque.

166. Cf. Diod. II, 58, 2-4. Chez Évhémère les bêtes sauvages ont certes un aspect extraordinaire et des forces étonnantes, mais ce sont des animaux existant réellement : éléphants, lions, panthères, antilopes (cf. Diod. V, 45, 1)!

167. Chez Iamboulos, par exemple, les hommes vivent jusqu'à cent cinquante ans (Diod. II, 57, 4) et possèdent une double langue qui leur permet de discuter avec deux interlocuteurs en même temps (Diod. II, 56, 5). 
temps oubliée, et dont la réinscription sur les cartes de la terre invite à relire l'histoire du passé mythique de l'ensemble du monde connu. La Panchaïe a été conçue par Évhémère comme le nouveau centre religieux de l'univers, dont le lecteur pouvait percevoir les manifestations concrètes au quotidien, puisque tout l'encens consommé dans le monde provenait de l'île Sacrée, sorte d'avant-poste de l'archipel panchaïen. Le choix des Crétois comme classe dirigeante était aussi une manière de rattacher la Panchaïe au reste du monde, puisque, selon la légende, il existait un tombeau de Zeus en Crète: les théories de la stèle trouvaient ainsi un ancrage dans la réalité. À la fois lointaine et proche, ancienne et à l'image du monde contemporain, la Panchaïe jouait un rôle de passerelle entre présent hellénistique et passé mythique revisité, si bien que le récit de voyage en ces îles du bout du monde était une parfaite introduction à la lecture de l'histoire divine qu'Évhémère présentait dans la suite de son ouvrage. La I $\varepsilon \rho \dot{\alpha}$ 'A $v \alpha \gamma \rho \alpha \phi \eta$ ' est ainsi un excellent exemple de la manière dont un auteur peut réemployer les traits traditionnels de l'imaginaire utopique et, en les réorganisant autour d'enjeux propres à son ouvrage - en l'occurrence ici la légitimation d'une théorie religieuse - faire œuvre pleinement originale.

Sébastien MONTANari

Université François Rabelais - Tours 


\section{Références bibliographiques}

\section{Auteurs anciens}

Aristote (Aubonnet 1986), Politique, vol. III (1 ${ }^{\text {re }}$ partie), livre VII, J. Aubonnet (éd. et trad.), Paris, Les Belles Lettres (CUF).

Atrúnée (Kaibel 1962), Deipnosophistae, vol. III, libri XI-XV, G. Kaibel (éd.), Stuttgart, Teubner (BT).

Cicéron (Pease 1955), De natura deorum libri III, vol. I, A.S. Pease (éd.), Cambridge (Mass.), Harvard University Press.

Diodore de Sicile (Vogel 1964), Bibliotheca Historica, vol. II, F. Vogel (éd.), Stuttgart, Teubner (BT).

Eusèbe de Césarée (des Places \& Sirinelli 1974), La Préparation évangélique, livre I, É. des Places, J. Sirinelli (éd. et trad.), Paris, Cerf (SC; 206).

Hérodote (Legrand 1983), Histoires, P.-E. Legrand (éd. et trad.), Paris, Les Belles Lettres (CUF).

Historiens d'Alexandre (Auberger 2001), J. Auberger (trad.), Paris, Les Belles Lettres (Fragments).

Homère (Bérard 1953), L'Odyssée, vol. I, livres I-VII, V. Bérard (éd. et trad.), Paris, Les Belles Lettres (CUF).

Hygin (Boriaux 1997), Fables, J.-Y. Boriaux (éd. et trad.), Paris, Les Belles Lettres (CUF).

LaCtance (Monat 1986), Institutions divines, livre I, P. Monat (éd. et trad.), Paris, Cerf (SC; 326).

Platon (Rivaud 1956), Timée-Critias, A. Rivaud (éd. et trad.), Paris, Les Belles Lettres (CUF). Pline l'Ancien (Schilling 1977), Histoire naturelle, livre VII, R. Schilling (éd. et trad.), Paris, Les Belles Lettres (CUF).

\section{Études}

BiCHLER R. (1984), «Zur historischen Beurteilung der griechischen Staatsutopie », GB, 11, p. 179-206.

BLock R. (de) (1876), Évhémère, son livre et sa doctrine, Mons, H. Manceaux.

Chamoux F. (1987), «Diodore de Sicile et la Libye», QAL, XII, p. 57-65.

DochHorn J. (2000), «Ein 'Insschriftenfund' auf Panchaia: Zur Hiera Anagraphè des Euhemeros von Messene », in Internationales Josephus-Kolloquium (Aarhus, 1999), J.U. Kalms (éd.), Münster, Lit (Münsteraner Judaistische Studien ; 6), p. 265-295.

Ferguson J. (1975), Utopias of the Classical World, Ithaca, Cornell University Press.

Gabrielsen V. (2004), "La piraterie et le commerce des esclaves », in Le Monde hellénistique. Espaces, sociétés, cultures 323-31 av. J.-C., A. Erskine (dir.), Rennes, Presses universitaires de Rennes, p. 495-512. 
Holzberg N. (1996), "Utopias and fantastic travel», in The Novel in the Ancient World, G. Schmeling (éd.), Leyde, Brill (Mnemosyne, Supplem.; 159), p. 621-632.

Mossé C. (1969), «Les utopies égalitaires à l'époque hellénistique », RH, 93, p. 297-308.

Mund-Dopchie M., Vanbaelen S. (1989), «L'Inde dans l'imaginaire grec », LEC, 57, p. 209226.

Peterson K. (2001), Living on the edge: the travel narratives of Euhemeros, Iamboulos and Lucian, Durham (North Carolina), Duke University.

Thraede K. (1965), «Euhemerismus », RLAC, VI, 46, p. 877-890.

Winiarczy M. (2002), Euhemeros von Messene. Leben, Werk und Nachwirkung, Munich Leipzig, K. G. Saur (Beiträge zur Altertumskunde ; 157).

Zumschlinge M. (1976), Euhemeros, Staatstheoretische und staatsutopische Motive, Phil. Diss., Bonn. 